\title{
Control of diamond heteroepitaxy on nickel by optical reflectance
}

\author{
P. C. Yang, R. Schlesser, C. A. Wolden, W. Liu, R. F. Davis, and Z. Sitar \\ Department of Materials Science and Engineering, North Carolina State University, Raleigh, \\ North Carolina 27695-7919
}

J. T. Prater

Army Research Office, Research Triangle Park, North Carolina 27709

(Received 3 February 1997; accepted for publication 1 April 1997)

\begin{abstract}
Real time in situ laser reflectometry was used to investigate changes in surface morphology observed during the nucleation of oriented diamond on $\mathrm{Ni}$ in a hot filament chemical vapor deposition reactor. Characteristic features observed in the intensities of reflected and scattered light were interpreted by comparison with scanning electron micrographs of the diamond seeded substrates quenched at sequential stages of the process. Based on this analysis, a process was developed in which the scattered light signal was used as a steering parameter. Using this process, oriented nucleation and growth of diamond on $\mathrm{Ni}$ can be repeatedly achieved. (C) 1997 American Institute of Physics. [S0003-6951(97)02622-3]
\end{abstract}

The growth of heteroepitaxial films represents an important step toward the attainment of large-area, device-quality diamond. The high surface energy of diamond and interfacial stress between diamond films and nondiamond substrates are believed to be primary obstacles to the formation of oriented, two-dimensional diamond nuclei. Nickel is one of a few materials that has a small lattice mismatch with diamond ( $a$ $=3.52 \AA$ for Ni versus $a=3.56 \AA$ for diamond). It has been known for decades that $\mathrm{Ni}$ is an effective solvent catalyst for diamond crystallization under high-pressure and hightemperature (HPHT) conditions. ${ }^{1}$ Some success has been attained using $\mathrm{Ni}$ as a substrate for diamond growth by lowpressure chemical vapor deposition (CVD). Sato et al. ${ }^{2}$ reported that both (111) and (100) oriented diamond nuclei could be grown on $\mathrm{Ni}$ substrates, but the overall percentage of oriented nuclei was rather low. We have observed that under certain conditions, oriented diamond nuclei precipitate from a supersaturated $\mathrm{Ni}-\mathrm{C}-\mathrm{H}$ solution., ${ }^{3,4}$ A similar phenomenon has been observed for the heteroepitaxial growth of diamond on (111) platinum by Kobashi and Tachibana and co-workers. ${ }^{5}$ Roy and co-workers ${ }^{6}$ also reported on precipitation of diamond particles from different mixtures of diamond and metal powders. Despite these successes, the mechanism by which oriented nuclei are formed is not understood. Also, the high catalytic activity of Ni for hydrocarbon decomposition and high solubility of carbon in Ni often lead to either graphite deposition or no growth if the process is not properly controlled. In order to overcome these difficulties, we developed a multistep seeding and growth process to obtain oriented diamond on nickel. ${ }^{3,4}$ The process is very sensitive to the substrate temperature and requires accurate timing of both the nucleation and growth steps. Accurate process control is requisite to initiate diamond growth and to suppress the deposition of graphitic products. It has been observed that the surface morphology changes dramatically during the nucleation process and that in situ monitoring of the surface structure can provide valuable feedback for process control.

In this letter, we report on the use of optical reflectometry to monitor changes in surface morphology in real time throughout the diamond seeding, annealing, and growth steps. Reflected and scattered light signals were correlated with surface structures by comparison with scanning electron micrographs (SEMs) of samples that were quenched at different stages of the nucleation process. Based on these studies, a process control procedure was developed where experimental parameters were adjusted as a function of the scattered light signal. This control has significantly improved both the reproducibility and overall quality of the oriented diamond films on Ni substrates.

The growth experiments were performed in a hot filament CVD diamond growth system that has been described previously. ${ }^{3,4}$ The chamber was modified to allow in situ optical measurements of reflective and scattering properties of the sample surface. Figure 1 shows the setup of the optical monitoring system. A He-Ne laser $(\lambda=633 \mathrm{~nm})$ illuminated the sample under normal incidence. A prism was used as a semitransparent beam splitter, which directed the reflected beam from the sample to a photodiode (detector 1). Light scattered from the sample surface was detected by a second photodiode positioned at an angle of $5^{\circ}$ from the surface normal. The field of view for detector 2 was defined by a pair of apertures. In order to discriminate between the probe beam and the intense light of the filament used for dissociation of $\mathrm{H}_{2}$, each detector was equipped with an optical inter-

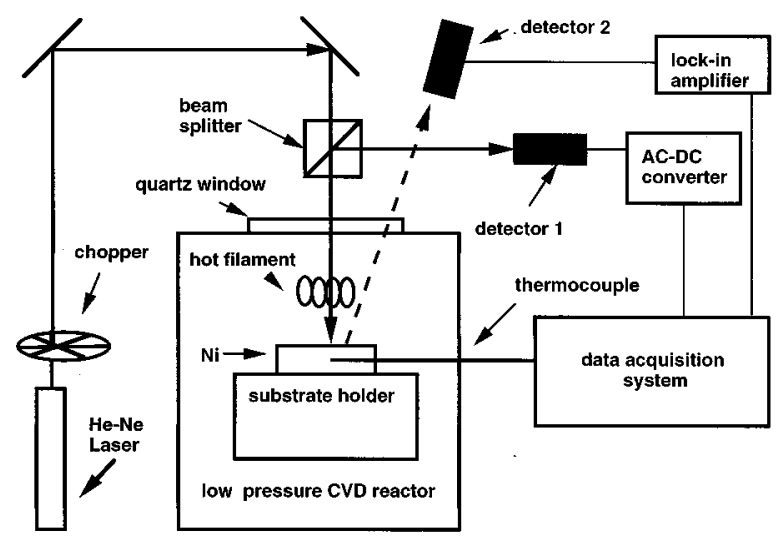

FIG. 1. Schematic of the hot-filament reactor equipped with optical diagnostics that was used for heteroepitaxial nucleation and growth of diamond on nickel. 


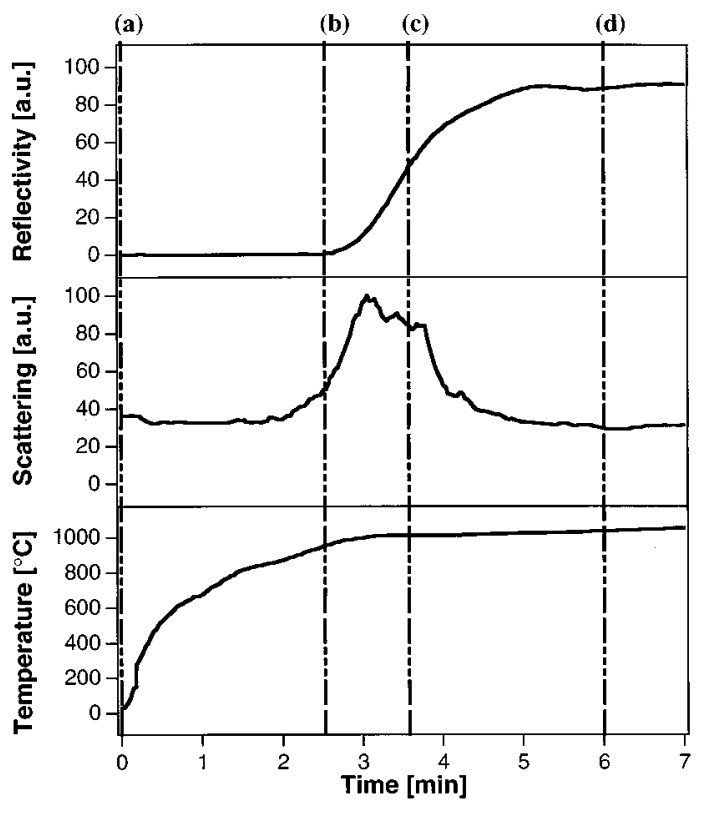

FIG. 2. Typical changes in the intensities of reflected light, scattered light, and substrate temperature during the nucleation process.

ference filter. Furthermore, the incident laser beam was mechanically chopped $(v=500 \mathrm{~Hz})$. The relatively strong reflected signal was processed by an ac-to-dc RMS converter, which suppressed the constant filament light contribution. A phase-sensitive lock-in amplifier was used to detect the weaker scattering signal. The substrate temperature was measured by a type $\mathrm{C}$ thermocouple imbedded $\sim 100 \mu \mathrm{m}$ beneath the substrate surface. All optical and temperature signals were recorded simultaneously by a Macintosh-based data acquisition system.

The experimental procedure used to generate oriented diamond nuclei consisted of three steps. First, a polished nickel substrate was seeded with a loosely applied layer of submicron diamond powder. Subsequently, the substrate was heated by the hot filament in a hydrogen environment to a relatively high temperature $\left(\sim 1100{ }^{\circ} \mathrm{C}\right)$ for a short annealing time $(3-10 \mathrm{~min})$ to produce a $\mathrm{C}$-saturated surface. Finally, the temperature was lowered to $\sim 900^{\circ} \mathrm{C}$ and methane was introduced to initiate gas phase growth of diamond.

Figure 2 shows typical changes in the intensities of reflected light, scattered light, and substrate temperature during the high-temperature anneal. Initially, the reflected light intensity was weak due to considerable scattering losses on the diamond seeds. The substrate temperature was rapidly increased to above $1000{ }^{\circ} \mathrm{C}$, eventually saturating at $1050{ }^{\circ} \mathrm{C}$. Simultaneously, a transient peak was observed in the scattered light intensity accompanied by a rapid increase in the reflected light signal. At the high temperatures, the reflected light reached a maximum, while the scattered light signal dropped back to its initial value. The typical changes in the optical signals (i.e., the rapid increase in reflectivity, accompanied by a transient peak in scattered intensity) were found to be reproducible from run to run and, therefore, provided a

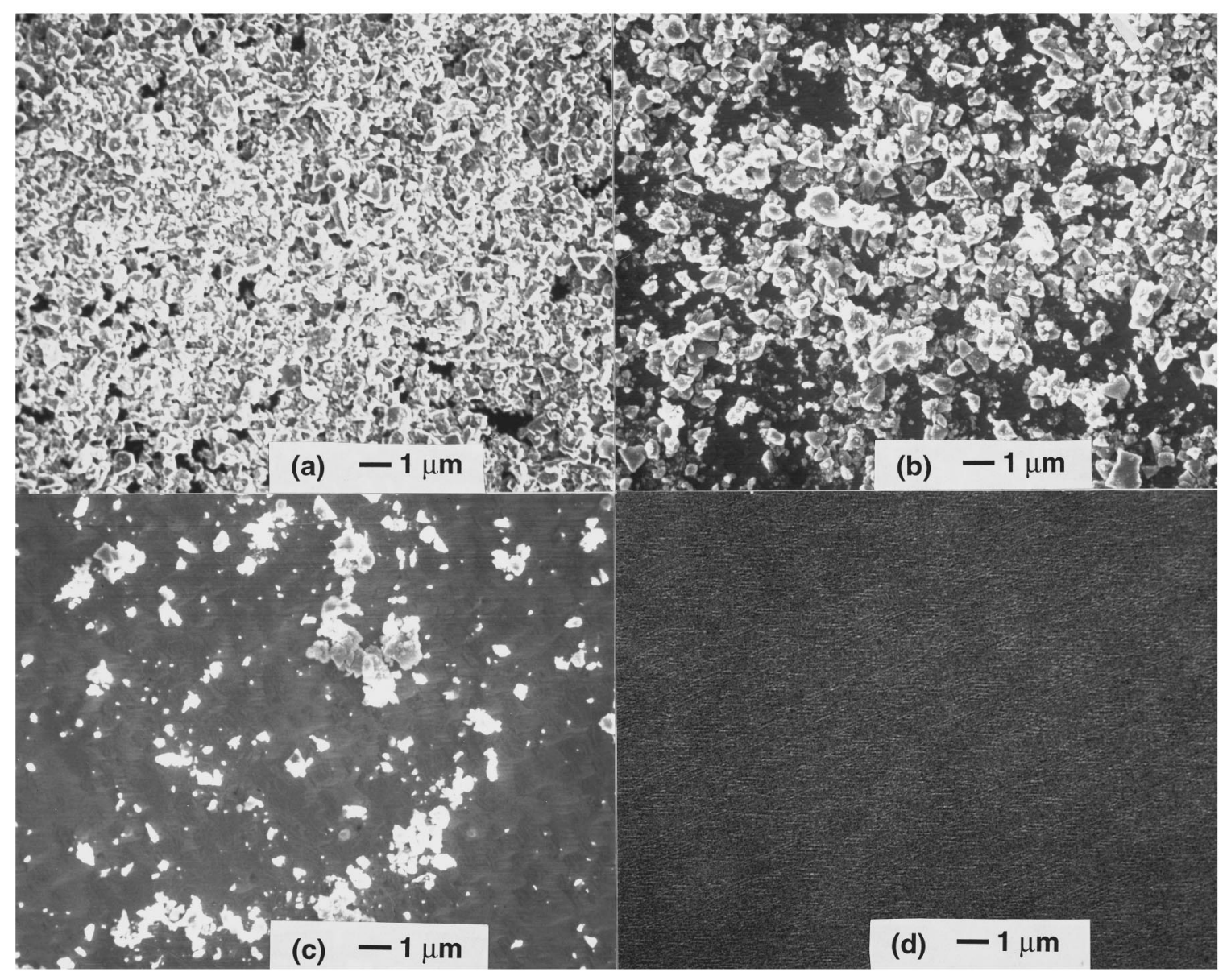

FIG. 3. Surface morphologies of samples quenched at four different stages of the nucleation process, as indicated in Fig. 2. 

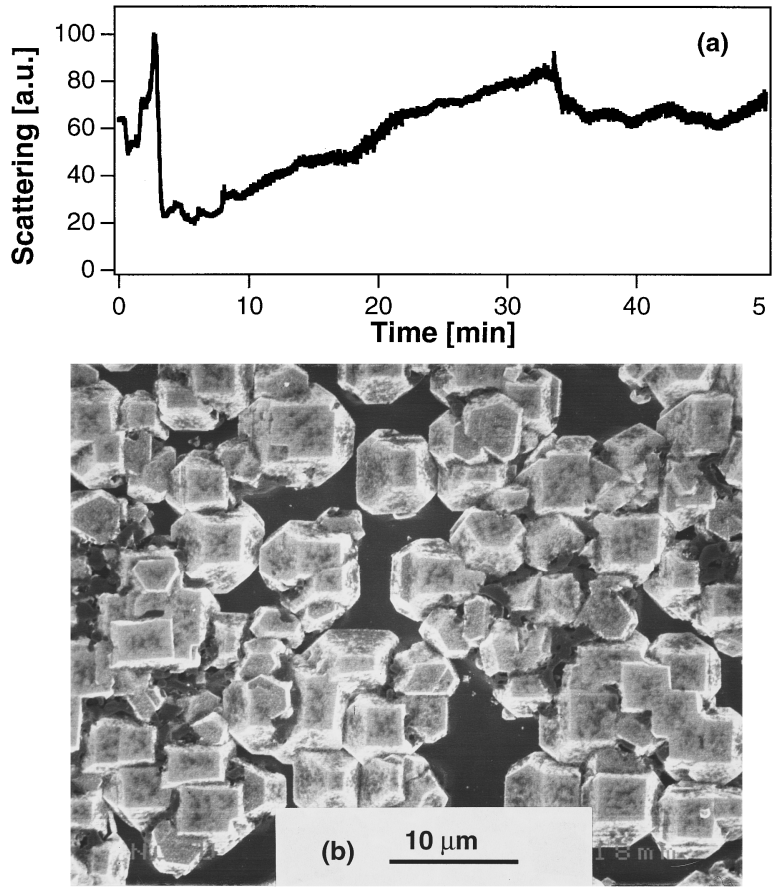

FIG. 4. (a) Changes in the scattered light intensity throughout the annealing and growth process, and (b) SEM micrograph of resulting diamond film on $\mathrm{Ni}$.

powerful tool for monitoring and controlling the pregrowth stage of the growth process.

In order to correlate the observed optical signals with surface features, samples were quenched at four stages [labeled (a), (b), (c), (d)] of the process as indicated in Fig. 2. The quenched samples were analyzed by scanning electron microscopy and the results are shown in Fig. 3. In the beginning (a), the Ni substrate was covered by a layer of diamond seeds produced by the seeding step. At time (b), as the reflected light intensity began to increase, the layer of diamond seeds was no longer continuous or uniform in size. A large fraction of the diamond seeds were either dissolved into the substrate or were etched away by atomic hydrogen. At time (c), when the scattered light was most intense, the population of the diamond seeds was greatly reduced and the size had become more uniform ranging from 100 to $200 \mathrm{~nm}$. At time (d), the reflected light had saturated at a maximum intensity and the scattered light intensity decreased. At this point, all the diamond seeds had disappeared from the surface and, upon quenching, and ordered crystalline surface morphology was obtained [Fig. 3 (d)].

Based on its sensitivity to changes in surface morphology, the scattered light signal was chosen as the indication of the time to change the experimental conditions from the pretreatment stage to the normal growth stage. In order to achieve oriented diamond nucleation, the diamond seeds must be fully reacted with the nickel substrate. This was indicated by a rapid decrease in the scattered light intensity after the peak (Fig. 2). Because carbon would further diffuse into the substrate, maintaining a high temperature for a long time would reduce the carbon rich environment at the surface and subsequently lower the nucleation density. Therefore, following the drop in the scattered light signal, the substrate temperature was lowered from 1050 to $900{ }^{\circ} \mathrm{C}$ and $0.5 \%$ $\mathrm{CH}_{4}$ was added to the $\mathrm{H}_{2}$ gas flow. The change in the scattered light signal during a complete process is shown in Fig. 4(a). The steady increase in the scattered light signal after lowering of the substrate temperature was attributed to the nucleation and growth of diamond. After $5 \mathrm{~h}$ of growth at $900{ }^{\circ} \mathrm{C}$, oriented diamond was obtained, as shown in Fig. 4(b).

In conclusion, optical reflectometry has been demonstrated to be a technique sensitive to morphology changes associated with the seeding and annealing process of diamond on Ni substrates. It was found that a transient increase in the scattered light intensity can be used as a control parameter. Use of scattered light as a process control resulted in reproducible production of oriented diamond on nickel.

The financial support of the Army Research Office under Grant No. DAAH04-93-D-0003 is gratefully acknowledged.

${ }^{1}$ R. H. Wentorf, Jr., Adv. Chem. Phys. 9, 365 (1965).

${ }^{2}$ Y Sato, H. Fujita, T. Ando, T. Tanaka, and M. Kamo, Philos. Trans. R. Soc. London, Ser. A 342, 225 (1993)

${ }^{3}$ P. C. Yang, W. Zhu, and J. T. Glass, J. Mater. Res. 8, 1773 (1993).

${ }^{4}$ P. C. Yang, W. Zhu, J. T. Glass, U.S. Patent No. 5298286 (1994).

${ }^{5}$ T. Tachibana, Y. Yokota, K. Nishimura, K. Miyata, K. Kobashi, and Y. Shintani, Diam. Relat. Mater. 5, 197 (1996).

${ }^{6}$ H. S. Dewan, D. Ravichandran, J. P. Cheng, W. R. Drawl, K. A. Cherian, and R. Roy, Proceedings of the Applied Diamond Conference 1995, Gaithersburg, MD, p 387. 